\title{
Islet inflammation in type 2 diabetes and physiology
}

\author{
Kosei Eguchi' and Ryozo Nagai \\ 'Department of Cenetics and Complex Diseases and Sabri Ülker Center, Harvard T.H. Chan School of Public Health, Boston, Massachusetts, USA. Jlichi Medical University, Tochigi, Japan.
}

\begin{abstract}
The finding of islet inflammation in type 2 diabetes (T2D) and its involvement in $\beta$ cell dysfunction has further highlighted the significance of inflammation in metabolic diseases. The number of intra-islet macrophages is increased in T2D, and these cells are the main source of proinflammatory cytokines within islets. Multiple human studies of T2D have shown that targeting islet inflammation has the potential to be an effective therapeutic strategy. In this Review we provide an overview of the cellular and molecular mechanisms by which islet inflammation develops and causes $\beta$ cell dysfunction. We also emphasize the regulation and roles of macrophage polarity shift within islets in the context of T2D pathology and $\beta$ cell health, which may have broad translational implications for therapeutics aimed at improving islet function.
\end{abstract}

\section{Introduction}

Metabolic syndrome comprises a cluster of diseases associated with excess nutrition and insufficient physical activity. Studies over the last two decades have shown that chronic inflammation is a common and potentially unifying mechanistic cause of these diseases (1-4). Inflammation can be viewed as an evolutionarily selected protective response enabling the host organism to cope with stresses from external factors, and can be classified as acute or chronic (5). Acute inflammation is characterized by prominent local and systemic signs, as well as infiltration of the affected area by immune cells, mainly neutrophils. By contrast, chronic inflammation is characterized by less prominent local and systemic signs, enhanced tissue injury and fibrosis, and infiltration of the affected area mainly with monocytes/macrophages and lymphocytes (5).

Inflammation in the context of metabolic syndrome largely exhibits the characteristics of chronic inflammation and is thus often accompanied by tissue infiltration by monocytes/macrophages $(6)$ and lymphocytes $(7,8)$. Type 2 diabetes (T2D) is a common and serious complication of metabolic syndrome, and although the disease can exist in isolation, many T2D patients meet the diagnostic criteria for metabolic syndrome (9). Given that chronic metabolic stress induced by excess nutrition was not a driving force during evolution, it is perhaps not surprising that inflammation in response to this stress eventually results in deleterious effects on tissue function and contributes to T2D pathology.

Insulin resistance and $\beta$ cell dysfunction are the two major components of T2D pathology, and $\beta$ cell function starts to decline even before the onset of impaired glucose tolerance (10, 11). Histologic changes characteristic of inflammation occur within the islets of T2D subjects, including immune cell infiltration (12-16), amyloid deposition $(14,17,18)$, cell death, and fibrosis $(18,19)$. These reports suggest inflammation is involved in $\beta$ cell dysfunction, though inflammatory pathologic changes have

Conflict of interest: The authors have declared that no conflict of interest exists. Reference information: / Clin Invest. 2017;127(1):14-23. doi:10.1172/JCI88877. been observed in only a portion of T2D patients, suggesting that islet inflammation and its contribution to T2D pathology may vary among patients $(12-14,18)$.

Several rodent experimental models (20-23) as well as observations in humans (12-14) have made it clear that macrophages play a key role in the islet inflammation seen in T2D. The most well-studied mechanism by which islet macrophages cause $\beta$ cell dysfunction is through secretion of IL- $1 \beta$, and it has been demonstrated that interference with the IL- 1 pathway relieves T2D and restores $\beta$ cell function in both rodents $(24,25)$ and humans $(26-28)$. Factors that stimulate islet macrophages to secrete IL-1 $\beta$ in vivo include human islet amyloid polypeptide (hIAPP) (20, 23), palmitate (21), and endocannabinoid (22). The possibility that other immune cell types are involved in islet inflammation in T2D remains to be confirmed; while one study reported an increase in the B cell number (15), other groups have not observed changes in the number of immune cell types including neutrophils, lymphocytes, and mast cells (refs. 12$16,29,30$, and Table 1). It has also been suggested that some T2D patients develop islet autoimmunity during the course of disease and that contributes to $\beta$ cell functional decline (31).

There is significant heterogeneity among macrophages in terms of both their function and origin in vivo, and the transition between functional states occurs along a continuum regulated by the microenvironment, especially in the context of sterile inflammation (3234). Using the concept of M1-like and M2-like polarization of macrophages to describe an essentially heterogeneous population of tissue macrophages is a simplified operational framework (35). During islet inflammation, overall macrophage polarity shifts toward the proinflammatory classically activated M1-like phenotype, and this shift has been shown to contribute to $\beta$ cell dysfunction in T2D mouse models (21-23). Additionally, alternatively activated M2-like macrophages, which include macrophages with antiinflammatory (36), pro-fibrosis (37), and pro-angiogenic (38) phenotypes, were recently found to be the key mediators of $\beta$ cell proliferation during both development $(39,40)$ and adulthood $(41-44)$ in mice.

In this Review we present an overview of the links between islet inflammation and $\beta$ cell dysfunction in T2D, with a focus on the regulation of islet macrophage polarity through commu- 
Table 1. Analysis of immune cells in islets of T2D subjects

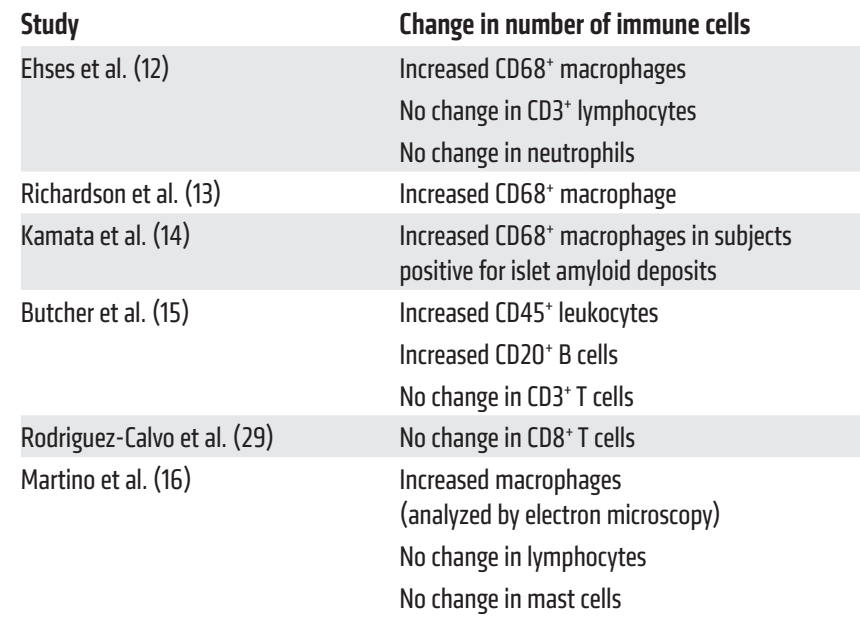

nications among multiple cell types within the islet and its roles in T2D pathology and physiology. From this viewpoint we argue that immune cell function within islets is seamlessly regulated and thus plays an indispensable role in maintaining $\beta$ cell health as well as serving as a primary source of proinflammatory cytokines that cause $\beta$ cell dysfunction in T2D. Expanding our understanding of the cells that mediate islet inflammation may have broad translational implications for therapeutics to improve islet function.

\section{Evidence for the involvement of islet inflammation and islet macrophage polarity shift in T2D}

Initial evidence of immune cell infiltration of the pancreatic islets in T2D came from immunostaining analyses showing increased numbers of islet macrophages in rodent T2D models (the GotoKakizaki [GK] rat and the $d b / d b$ [leptin-deficient] mouse; refs. 12, $45)$, as well as in human T2D patients $(12,13)$. Additionally, microarray profiling of human $\beta$ cells obtained through laser-capture microdissection revealed a three-fold increase in the expression of chemokines CCL2 and CCL13 in T2D subjects compared with controls, providing molecular evidence of immune cell infiltration and islet inflammation in human T2D patients (46). These results suggest that islet inflammation in T2D is mainly driven by innate immunity, whereas inflammation in type 1 diabetes (T1D) is known to be mainly driven by adaptive immunity $(47,48)$. Several studies also showed that prolonged exposure to high glucose levels causes $\beta$ cells to secrete IL-1 $\beta$, which contributes to islet inflammation in human islets in vitro (49) and in mice (50).

Given the heterogeneity of macrophages (32) and the importance of macrophage polarization in the pathology of obese adipose tissue (AT) (6), we asked whether there are multiple subtypes of macrophages within the islets and analyzed the extent to which their characteristics are altered in T2D islets. Compared with islets from control $d b /+$ mice, islets from $d b / d b$ mice expressed several-fold higher levels of such chemokines and cytokines as Ccl2, Cxcl1, Il1b, and Tnf. Flow cytometric (FCM) analysis demonstrated that islets from two murine models of T2D, obese $d b / d b$ mice or KKAy mice (mouse model described in ref. 51), contain more $\mathrm{CD} 11 \mathrm{~b}^{+}$Ly- $6 \mathrm{C}^{+}$macrophages without a significant change in

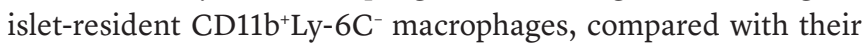
respective controls (21). We observed that $C D 11 b^{+} L y-6 C^{+}$cells from islets of obese mice do not express the cell surface proteins CD206 and CD301, which are characteristic of M2-like alternatively activated macrophages, and exhibit greater expression of Illb and Tnf and lower expression of Il1O than islet-resident CD11b ${ }^{+}$Ly- $6 \mathrm{C}^{-}$macrophages. These findings demonstrate that macrophages shift to an inflammatory M1-like phenotype within the islets of at least two different T2D mouse models (Figure 1). Another report found that M1-like $\mathrm{CD} 68^{+} \mathrm{F} 4 / 80^{-}$macrophages are present only in the islets of $d b / d b$ mice and islet-resident M2-like CD206-expressing $\mathrm{CD} 68^{+} \mathrm{F} 4 / 80^{+}$macrophages in the islets of both $d b /+$ and $d b / d b$ mice, confirming that islet macrophages in $d b / d b$ mice undergo a shift in polarization to an M1-like phenotype (52). In contrast, one recent study described Illb and $\operatorname{Tnf}$ expression in non-diabetic islet-resident macrophages, although this study did not compare these macrophages with the macrophages of inflamed islets (53).

\section{Islet macrophages link $\beta$ cell dysfunction and islet inflammation}

The first in vivo evidence that islet inflammation plays a causative role in T2D was derived from a human clinical study on the effect of anakinra, a recombinant human IL-1 receptor antagonist (IL$1 \mathrm{Ra}$ ) that blocks both IL- $1 \alpha$ and IL-1 $\beta$ signaling, in 70 patients with T2D (26). Thirteen weeks of treatment with anakinra significantly reduced serum IL-6 and C-reactive protein levels, improved glycemia and C-peptide secretion, and reduced the serum proinsulin/ insulin ratio without significantly affecting homeostatic model assessment of insulin resistance (HOMA-IR). These effects of anakinra on insulin secretion were also seen in prediabetic patients with impaired glucose tolerance (54). Further, studies utilizing multiple mAbs against IL- $1 \beta$ confirmed the contribution of IL- $1 \beta$ signaling to systemic inflammation, glycemic control (55), and $\beta$ cell dysfunction $(27,28)$ in T2D $(56,57)$. A dose-escalation study of govekizumab, a recombinant humanized mAb against IL-1 $\beta$, demonstrated that an intermediate dose $(0.03-0.1 \mathrm{mg} / \mathrm{kg})$ but not a high dose $(>0.3 \mathrm{mg} / \mathrm{kg})$ of gevokizumab significantly improved glycated hemoglobin $\mathrm{A} 1 \mathrm{C}$ (HbA1C), suggesting the effects of IL-1 $\beta$ in T2D are multifaceted (see below) (27). For greater mechanistic insight into the role of IL-1 signaling in $\beta$ cell dysfunction in T2D, IL-1Ra was administered to mice fed a high-fat diet (HFD) (25) and to GK rats (24). Ehses and colleagues clearly showed that treating GK rats with IL-1Ra resulted in decreased hyperglycemia that was accompanied by increased insulin mRNA levels within islets, reduced serum proinsulin/insulin ratios, reduced macrophage infiltration into islets and mRNA levels of chemokine/proinflammatory cytokine within islets, and ameliorated insulin resistance (24). There was no change in islet mass with IL-1Ra treatment $(24,25)$, suggesting that a functional decline of $\beta$ cells rather than an apoptotic effect is the primary mechanism by which islet inflammation decreases insulin secretion in T2D (Figure 1). This finding is consistent with the earlier observation that in T2D individuals, islets containing increased numbers of macrophages did not contain higher numbers of TUNEL-positive cells and macrophages were not observed in proximity to apoptotic 


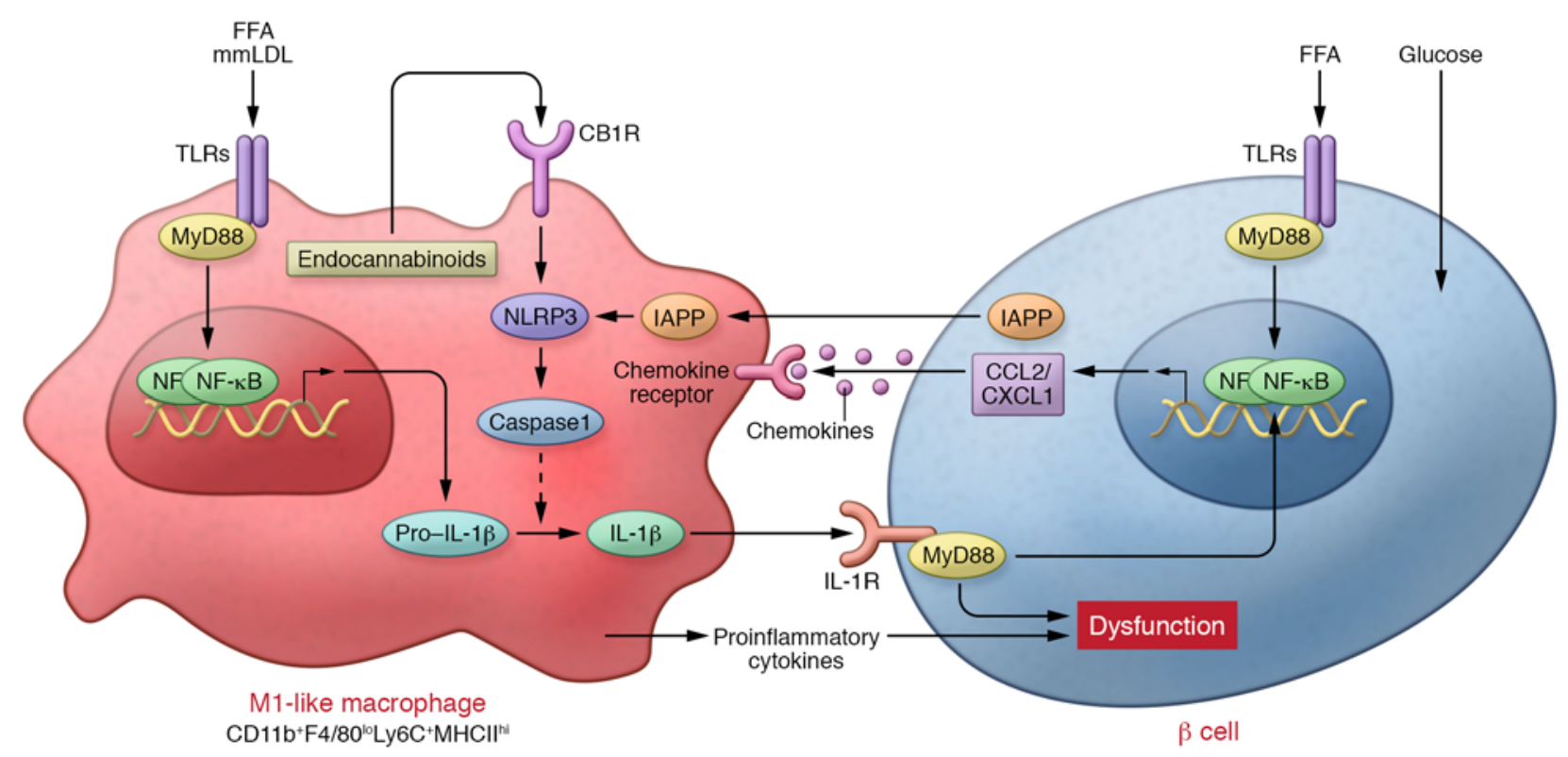

Figure 1. M1-like polarization of islet macrophages plays important roles in islet inflammation and $\beta$ cell dysfunction in T2D. The communication between islet macrophages and $\beta$ cells via hIAPP, chemokines (e.g., CCL2 and CXCL1), and proinflammatory cytokines (e.g., IL-1 1 ) initiate and amplify the M1-like polarity shift of islet macrophages and islet inflammation. The inflammasome/IL-1 $\beta$ pathway in islet macrophages is a common pathway that causes $\beta$ cell dysfunction within inflamed islets in T2D.

$\beta$ cells (12). This may be an important distinction from the effect of islet inflammation in T1D, where the primary mechanism of disease is the autoimmune destruction of $\beta$ cells (47).

The actions of macrophages leading to $\beta$ cell dysfunction in obese mice were further interrogated using clodronate liposomemediated suppression of macrophage recruitment to islets in $d b / d b$ and KKAy mice. In both models, mice treated with clodronate liposomes showed improved glucose tolerance with improved insulin secretion in an oral glucose tolerance test (OGTT), and islets isolated from these mice exhibited increased glucose-stimulated insulin secretion (GSIS) as compared to the blunted GSIS seen in islets from mice treated with empty liposomes. Levels of pancreatic and duodenal homeobox-1 $(P d x 1)$ and insulin mRNA were also increased in islets from clodronate liposome-treated mice (21). These data clearly demonstrate the causative involvement of islet inflammation and macrophage infiltration in $\beta$ cell failure in $\mathrm{T} 2 \mathrm{D}$ rodent models (Figure 1).

\section{Mechanisms of islet inflammation and inflammation-induced $\beta$ cell dysfunction}

The aforementioned studies support the therapeutic strategy of targeting islet inflammation to ameliorate T2D. To gain mechanistic insight into the pathophysiology of islet inflammation, experiments using a specific factor that initiates inflammation have several advantages. These include the ability to set up in vitro preparations to dissect the complex communication between multiple cell types within islets, easy utilization of mutant mice, and the ability to set up shortterm in vivo models to eliminate confounding effects from insulin sensitization due to systemic antiinflammatory manipulations. With these advantages in mind, in the next section we review reports analyzing islet inflammation induced by specific stimuli, including hIAPP, saturated free fatty acids (FFAs), and endocannabinoids.
Islet inflammation induced by TLR activation in T2D. In determining the mechanisms by which inflammation is induced in obese AT, the roles of pattern-recognition receptors, including TLRs (58, 59) and NLRs (60) have been extensively analyzed. Several factors, including saturated FFAs, minimally modified LDL (mmLDL) cholesterol, advanced glycation end products of LDL cholesterol, and damage-associated molecular patterns (61) such as HSP60, S100 calcium-binding protein A8 (62), and high-mobility group $\mathrm{B}$ (HMGB) proteins (63) all reportedly activate TLRs in obese AT (64). Indeed, saturated FFAs have been reported to induce chemokine (Cxcl1 and $C c l 3)$ expression within islets in vitro $(12,46)$. Additionally, HMGB1 has been shown to activate the NF- $\kappa B$ pathway in isolated murine islets via TLR2 and TLR4 pathways (65). Furthermore, $\mathrm{Tlr} 2^{-/-}$mice fed a HFD for 20 weeks were protected from HFD-induced $\beta$ cell dysfunction and insulin resistance (66), indicating a critical role for the TLR2 pathway in islet inflammation.

Treatment of islets isolated from healthy human control subjects with palmitate, but not oleate or high glucose, increased secretion of cytokines and chemokines, including IL-6 and CXCL1, mimicking the inflammation seen in islets from T2D patients. Palmitate-induced expression of inflammatory genes (IL6 and IL8) in human islets was IL-1 dependent, as IL-1Ra abolished the effects of palmitate (46). Similarly, in both human and mouse islets, exposure to FFAs induced $I l 1 b$ and $C x c l 1$ expression and pro-IL-1 $\beta$ expression. This inflammatory response was partially inhibited by IL-1Ra (67). Consistently, exposure of MIN6 $\beta$ cells to culture medium conditioned by palmitate-treated macrophages, with or without addition of IL-1 $\beta$ - and TNF- $\alpha$-neutralizing antibodies, confirmed that those proinflammatory cytokines are produced by macrophages, not by $\beta$ cells, and that they promote $\beta$ cell dysfunction and enhance chemokine expression in $\beta$ cells (21). These reports demonstrate the ability of saturated FFAs to initiate an 


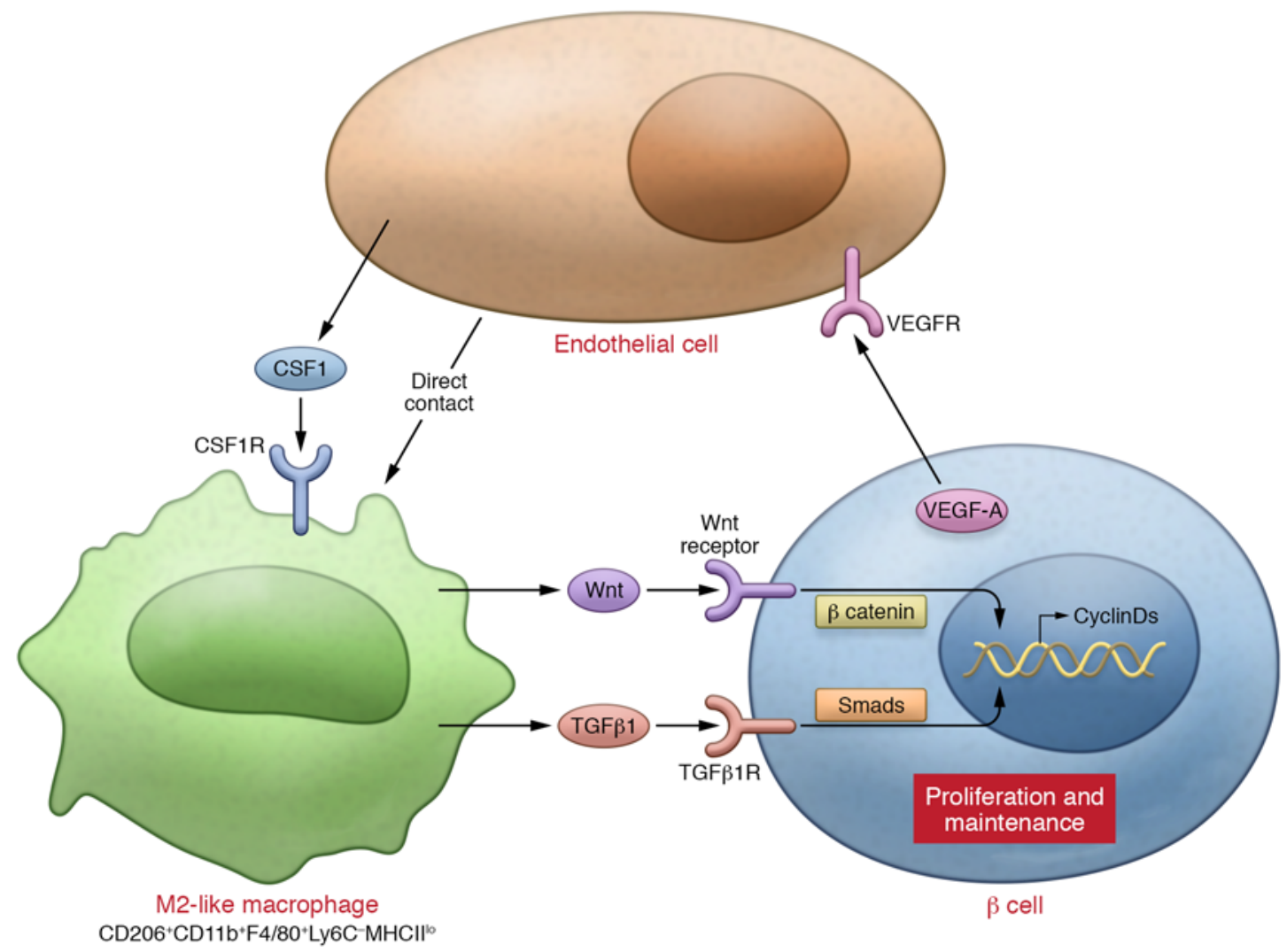

Figure 2. M2-like polarization of islet macrophages plays key roles in the proliferation and physiologic maintenance of $\beta$ cells. Multiple cytokines (e.g., TCF- $\beta 1$, VEGFA, and CSF1) secreted from multiple cell types within islets (e.g., macrophages, $\beta$ cells, and endothelial cells) form networks that contribute to a microenvironment that promotes M2-like polarization of islet macrophages. CSF1 signaling is a common mediator that maintains and promotes M2-like activation of both islet-resident and recruited macrophages. M2-like macrophages play an indispensable role in the establishment of the microenvironment necessary for $\beta$ cell health. CSF1R, CSF1 receptor.

inflammatory response in the islets, and also demonstrate a role for the autocrine or paracrine effects of IL-1 $\beta$. In vivo, short-term intravenous ethyl-palmitate infusion induced Ccl2, Cxcl1, Illb, and Tnf expression and reduced insulin and $P d x 1$ expression within islets, mimicking the observations from $d b / d b$ and KKAy mice (21) and individuals with T2D (46). FCM analysis of islets from these mice revealed that ethyl-palmitate infusion recruited $\mathrm{CD}_{11} \mathrm{~b}^{+}$ Ly- $6 \mathrm{C}^{+}$classically activated M1-like monocytes/macrophages to the islets. $\mathrm{Tlr} 4^{-/-}$and $\mathrm{Myd} 88^{-/-}$mice were completely protected from both M1-like macrophage recruitment and $\beta$ cell dysfunction. Bone marrow transplantation studies revealed that TLR4 in islet cells, but not in bone marrow cells, was required for M1-like macrophage recruitment. The short-term nature of the ethyl-palmitate experiment supports the causative contribution of M1-like macrophageinduced islet inflammation to palmitate-induced $\beta$ cell dysfunction without the confounding effect of insulin sensitization (21).

These reports demonstrate that TLR-mediated sensing of palmitate in $\beta$ cells is responsible for the initial chemokine secretion that induces macrophage recruitment. Thereafter, communication via proinflammatory cytokines and chemokines between M1-like macrophages and $\beta$ cells form a vicious cycle that amplifies islet inflammation (Figure 1). These findings are consistent with the previous finding that antagonizing IL-1 signaling inhibits both macrophage recruitment and $\beta$ cell dysfunction in GK rats (24).
Islet inflammation induced by IAPP in T2D. Amyloid deposits are commonly observed within islets from T2D patients (68). Islet amyloid deposits are composed of IAPP $(69,70)$, which is produced in $\beta$ cells and secreted in a monomeric form along with insulin (71). In pathologic conditions, secreted hIAPP is converted to a $\beta$-sheet structure and oligomerized $(72,73)$. The monomeric form of IAPP does not itself affect $\beta$ cell function, and only the aggregated form - particularly with non-fibrillar hIAPP oligomers, less with mature amyloid fibrils - exhibits toxicity toward $\beta$ cell function in vitro $(74,75)$. Given this fact, it is noteworthy that the oligomerization of IAPP differs among species. For example, human and feline IAPP can be oligomerized but rat and mouse IAPP cannot $(74,76)$. Thus hIAPP transgenic (hIAPP-Tg) mice (77) exhibit islet amyloid deposition, while transgenic mice expressing rat IAPP (rIAPP-Tg) do not (78). Further analysis of hIAPP-Tg mice revealed that dietary fat promotes islet amyloid formation and that one year on a HFD caused $\beta$ cell loss and impaired insulin secretion (79). Islets in hIAPP-Tg mice exhibited larger F4/80-positive areas and greater expression of chemokines (Ccl2 and Cxcl1) and macrophage markers (Emr1 and Itgax) than non-transgenic mice after one year on either a low-fat diet or HFD (80). Additionally, immunostaining studies revealed the presence of IAPP within the lysosomes of islet macrophages in hIAPP-Tg mice but not rIAPP-Tg mice, suggesting that macro- 


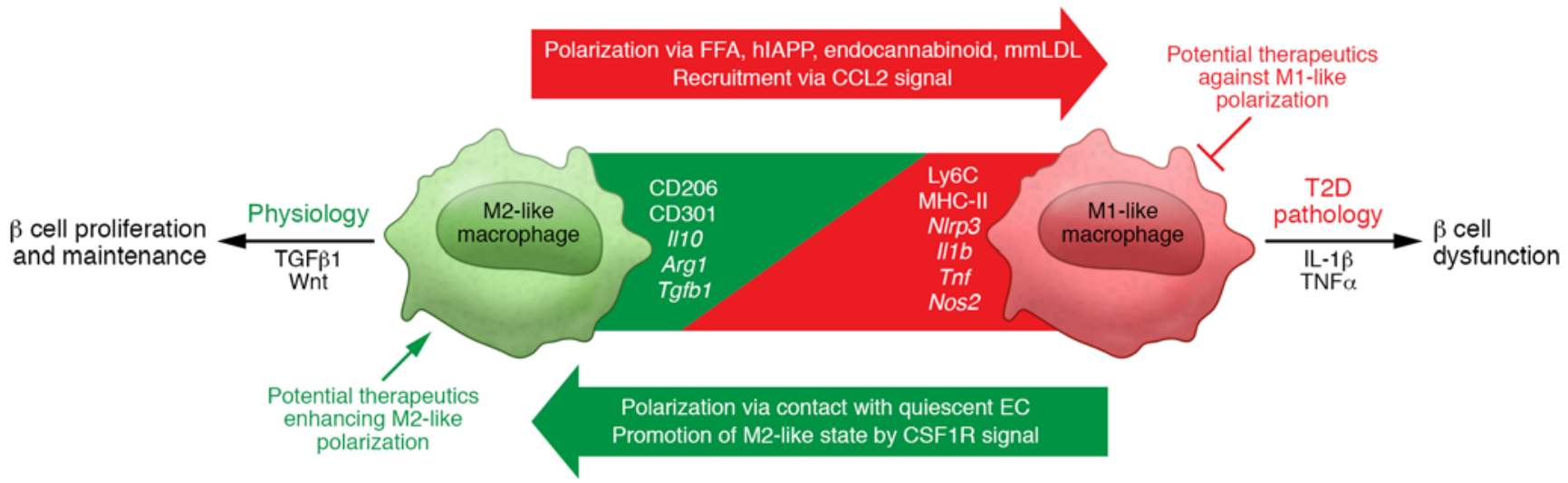

Figure 3. Overview of islet macrophage biology in the context of normal physiology and T2D pathology. M1-like macrophages are represented by surface expression of Ly6C and MHC-II and gene expression of NIrp3, I/1b, Tnf, and Nos2. M1-like macrophages are induced by such factors as FFAs, hIAPPs, and endocannabinoids, and at least some M1-like macrophages are recruited through CCL2 signaling. M2-like macrophages are represented by surface expression of CD206 and CD301 and gene expression of II10, Arg1, and Tgfb1. At least some M2-like macrophages self-renew or are recruited through CSF1 signaling. In the sterile islet inflammation observed in T2D, islet macrophages' polarization is seamlessly regulated as a continuum, rather than as distinct bimodal M1 and M2 polarization. Studies of the regulation of islet macrophage polarization, characterizations of subsets of heterogeneous islet macrophages, and analyses of the mechanisms by which these polarized macrophages exert their physiologic and pathologic effects on islet biology have the potential for translation to T2D therapeutics.

phages are involved in the toxic effects of islet amyloid deposition in T2D (78). Finally, in a human study using immunohistochemical analysis of islets, there were three-fold more $\mathrm{CD} 68^{+}$cells per unit area in islets from diabetic patients with islet amyloid deposition than in islets from non-diabetic patients or diabetic patients without amyloid deposition (14).

Masters and colleagues have presented a clear perspective on how hIAPP activates macrophages and induces islet inflammation (20). This group focused on the finding that inflammasome activation is the critical event in the initiation of islet inflammation by hIAPP, as hIAPP induced IL-1 $\beta$ but had no effect on IL- 6 or TNF secretion from mmLDL-primed dendritic cells in vitro. The significance of the inflammasome/IL-1 pathway to hIAPP-induced islet inflammation in vitro was further demonstrated by the finding that TNF secretion from macrophages and chemokine secretion from isolated islets induced by long-term stimulation with hIAPP was strongly suppressed by IL-Ra $(81,82)$. In vivo, IL-1Ra treatment improved glucose tolerance in recipients of hIAPP-Tg islets and conferred nearly complete protection from macrophage recruitment to the transplanted hIAPP-Tg islets (81). IL-1Ra also improved islet function without affecting insulin sensitivity in hIAPP-Tg KKAy mice (83).

In accordance with the earlier electron microscopic observation of amyloid within macrophage lysosomes (84), the involvement of phagolysosomes in hIAPP-mediated inflammasome activation was demonstrated in an experiment in which administration of the phagocytosis inhibitors cytochalasin $\mathrm{D}$ and bafilomycin A blocked inflammasome activation in macrophages in vitro (20). These mechanisms are consistent with the mechanisms of inflammasome activation in other amyloid-induced pathologies including Alzheimer's disease $(85,86)$. It was also shown that fibril aggregates accumulate within macrophage lysosomes, further supporting the involvement of hIAPP phagocytosis by macrophages (87). Immunostaining of islets from hIAPP-Tg mice fed a HFD for one year revealed that IL-1 $\beta$ colocalized with amyloid deposits and with macrophages, but not with $\beta$ cells. These studies show that macrophages are the source of IL-1 $\beta$ within islets both in vivo and in vitro (ref. 20 and Figure 1).

Several studies have sought to determine the identity of the priming factors that induce production of pro-IL-1 $\beta$ and the inflammasome component NLRP3 (88), which leads to inflammasome activation within T2D islets. Among several factors reported to stimulate TLR4 in metabolic syndrome $(58,89)$, Masters and colleagues confirmed that mmLDL cholesterol (90) can prime macrophages via TLR4 for inflammasome activation by hIAPP (20). More recent reports indicate that soluble hIAPP species produced during early hIAPP aggregation are themselves able to activate the TLR2/MyD88 pathway to prime macrophages $(82,91)$, and palmitate priming of inflammasomes has also been reported (92). Additionally, an independent group confirmed the presence of inflammasome markers (Nlrp3, Pycard, and Casp1) and inflammatory cytokines (Il1b, Tnf, and Il6) within islets of hIAPP-Tg mice fed a HFD (80).

Depletion of islet macrophages from hIAPP-Tg mice fed a HFD improved systemic glucose tolerance and GSIS of isolated islets, and almost completely blocked islet inflammation, despite greater deposition of amyloid within islets, demonstrating the causal role of islet macrophages in hIAPP-induced islet inflammation and $\beta$ cell dysfunction in vivo (23). hIAPP-induced IL-1 $\beta$ secretion and NLRP3/caspase-1 induction within isolated islets was completely abolished by clodronate liposome-mediated macrophage depletion (23), which demonstrates the contribution made by resident macrophages to inflammasome activation and IL-1 $\beta$ secretion induced by hIAPP in vitro. In this experiment, in contrast to complete inhibition of $I l 1 b$ expression, macrophage depletion led to an $80 \%$ reduction in hIAPP-induced $C c / 2$ expression, suggesting that nonphagocytic cells (likely $\beta$ cells) contribute to islet chemokine production (81). Although there was no obvious infiltration of islets by different macrophages subsets, FCM analysis showed that macrophages within islets from hIAPP-Tg 
mice fed a HFD express more CD11b, CD11c, and Ly-6C as well as more $I l 1 b$ mRNA and less $I l 10$ mRNA than macrophages within islets from wild-type mice fed a HFD. From these findings it was speculated that hIAPP aggregation may affect the differentiation state of monocytes entering islets and that hIAPP polarizes resident macrophages toward a proinflammatory M1-like phenotype (ref. 23 and Figure 1).

Islet inflammation induced by endocannabinoid activation of inflammasomes in islet macrophages. Cannabinoid receptors are expressed in both nervous systems and the peripheral tissues. The most potent endocannabinoids (i.e., endogenous agonist for the cannabinoid receptors) are the lipid ligands anandamide and 2-arachidonoylglycerol. Endocannabinoids play multiple roles, including the regulation of appetite and mood in the nervous systems and the regulation of energy homeostasis in peripheral tissues $(93,94)$. The cannabinoid 1 receptor (CB1R) antagonist ibipinabant reportedly attenuates $\beta$ cell loss in diabetic Zucker diabetic fatty (ZDF) rats (95). Investigation into this observation revealed a new mechanism for $\beta$ cell loss involving CB1R signaling in islet macrophages. Jourdan and colleagues first confirmed that treating ZDF rats with the non-brain-penetrant CB1R inverse agonist JD5037 (96) preserved pancreatic $\beta$ cell function and protected against $\beta$ cell loss (22). Immunohistochemical analysis of the isolated islets revealed that JD5037 reduced macrophage infiltration into islets. mRNA analysis demonstrated that JD5037 reversed the M1-like polarity shift of islet macrophages, characterized by decreased expression of Tnf, Nos2, Nlrp3, and Cnr1 and increased expression of Tgfb1, Il10, and Arg1 within ZDF islets. Moreover, CB1R and NLRP3 were expressed in $\mathrm{CD} 68^{+}$macrophages but not in insulin-producing cells. Macrophage depletion using clodronate liposomes demonstrated that macrophages are responsible for the increases in Cnr1, Nlrp3, Txnip, and Tnf expression and for the reduced insulin secretion capacity in ZDF rats. Additionally, the effect of macrophage CB1R on islet inflammasome activation and diabetes development was confirmed through macrophageselective knockdown of Cnr1 in ZDF rats. Finally, macrophage production of anandamide was stimulated by high glucose and palmitate in vitro. With these experiments, the authors demonstrated that in ZDF rats, autocrine activation of CB1R on macrophages by anandamide causes inflammasome activation and $\beta$ cell dysfunction and apoptosis (Figure 1).

\section{Beneficial and physiologic functions of islet macrophages}

The studies summarized above suggest there is therapeutic potential in targeting cytokines from islet macrophages as well as the mechanisms responsible for M1-like polarization of islet macrophages. However, the fact that lean Il1b-deficient mice exhibit glucose intolerance with reduced islet insulin and $P d x 1$ transcription (97) clearly indicates that these cytokines and the cells that secrete them also exert beneficial effects. Several analyses on the beneficial effects of low-concentration IL-1 $\beta$ on $\beta$ cells have been performed, including investigation into the mechanisms involving the Fas-FLIP pathway (97) and insulin granule docking to the plasma membrane (98). Further studies of the physiologic and beneficial functions of islet macrophages and cytokines will enhance the safety and efficacy of therapeutics that target M1-like polarization and cytokine secretion. Beyond that, these studies would aid in the development of new therapeutic strategies aimed at enhancing the beneficial aspects of macrophage functions.

It has long been known that macrophages are present within healthy islets (99) and are important for the expansion of $\beta$ cell mass $(39,40,100)$. These studies demonstrated the indispensable role of CSF1/CSF1R signaling from duct epithelial cells for islet-resident macrophage development and the essential contribution made by islet-resident macrophages to efficient $\beta$ cell proliferation during embryonic development and adulthood. Therefore, characterization of islet-resident macrophages has translational possibilities. Recent studies have shown that there are at least two distinct lineages of macrophages, and that lineage may affect their functional potential. The first macrophage lineage, which is derived from the yolk sac (YS), is established before the appearance of hematopoietic stem cells (HSCs) and persists as a population of $\mathrm{F} 4 / 80^{\text {bright }}$ macrophages that self-renew throughout adulthood in many tissues $(101,102)$. In some tissues, like intestine, YS-derived macrophages are replaced by HSC-derived cells; consequently, adult mice lacking CCR2, which is required for macrophage trafficking from the bone marrow, have markedly reduced tissue macrophages (103). The second macrophage lineage, which is derived from HSCs, gives rise to circulating monocytes that differentiate into $\mathrm{CD} 11 \mathrm{~b} \mathrm{~b}^{\text {hi }} \mathrm{F} / 8 \mathrm{O}^{\mathrm{lo}}$ macrophages, which are dependent on the transcription factor Myb. Analysis of mouse pancreas showed that pancreatic F4/80 bright macrophages found in proximity to $\beta$ cells at E16.5 are Myb independent, indicating that islet macrophages originate from the YS $(101,104,105)$. Recent studies also showed that islet macrophages are absent in Csf1 mutant op/op mice in adulthood, are self-maintained independently from bone marrow-derived circulating monocytes, have a low proliferation rate (53), and are not affected by Ccl2 and Ccr2 deficiency (21), characteristics that are consistent with a YS origin. In contrast, lineage-tracing studies have shown that islet macrophages exhibit high positivity for HSC progeny markers (53). Therefore, the origin of islet resident macrophages remains to be confirmed by further analyses.

Recent studies have indicated that macrophages also play an important role in the maintenance of $\beta$ cell mass and proliferation of $\beta$ cells in response to increased workload or damage to islets. In a pancreatitis model it was shown that CSF1R signaling in M2-like macrophages is important for $\beta$ cell proliferation and islet angiogenesis (41). In a study analyzing the streptozotocin model of diabetes, stromal cell-derived factor 1 recruited M2-like macrophages via CXCR4, after which these macrophages activated Wnt signaling in $\beta$ cells, making M2-like macrophages indispensable for $\beta$ cell replication (44). In the partial pancreatic ductal ligation (PDL) model, a well-established model of damage-induced islet proliferation, clodronate liposome-mediated macrophage ablation revealed the indispensability of $\mathrm{F} 4 / 80^{+}$macrophages recruited to islets for $\beta$ cell proliferation (42). The majority of the recruited macrophages were $\mathrm{CD} 206^{+} \mathrm{F} 4 / 80^{+}$macrophages that exhibited increased Arg1 expression and decreased Nos 2 expression, which is indicative of the alternatively activated M2-like phenotype. These macrophages contributed to the microenvironment necessary for $\beta$ cell proliferation by releasing TGF- $\beta 1$ (106), which induced SMAD7 in $\beta$ cells. SMAD7 promotes replication of pre-existing $\beta$ cells by increasing cyclin D1 and cyclin D2 and by inducing nuclear exclusion of p27 (ref. 42 and Figure 2). 
Recently, studies of VEGF in $\beta$ cells clarified the mechanisms underlying M2-like macrophage activation and the requirement for M2-like macrophages in $\beta$ cell proliferation in mice. It was previously shown that Vegfa deficiency in $\beta$ cells causes impaired islet vascularization and impaired insulin secretion (107), while Vegfa overexpression in $\beta$ cells results in islet hypervascularization, inflammation, and $\beta$ cell dysfunction (108). In a transient $\beta$ cell-specific Vegfa-overexpressing mouse model, which demonstrates intra-islet endothelial cell proliferation accompanied by macrophage recruitment and decreased $\beta$ cell proliferation and mass, withdrawal of the Vegfa overexpression stimulated $\beta$ cell proliferation. Inhibiting macrophage recruitment through partial bone marrow ablation revealed the importance of M2-like $\mathrm{CD} 45^{+} \mathrm{CD} 11^{+} \mathrm{Gr} 1^{-}$macrophage recruitment to islets for $\beta$ cell proliferation (109). Interestingly, it was reported that endothelial cells could provide a niche for M2-like macrophage polarization through their direct contact with macrophages and also by providing paracrine factors, including CSF1 (110). It therefore seems that quiescent endothelial cells provide the local environment that enables M2-like activation of macrophages, and the environment established by these endothelial cells and M2-like macrophages promotes $\beta$ cell proliferation (Figure 2 ).

To further analyze the origin and mechanisms of the M2-like macrophage activation required for $\beta$ cell proliferation, Van Gassen and colleagues utilized adoptive transfer of $\mathrm{GFP}^{+} \mathrm{CD} 11 \mathrm{~b}^{+} \mathrm{Ly} 6 \mathrm{C}^{\mathrm{h}}$ monocytes in a PDL model (111). This study demonstrated that circulating $\mathrm{CD} 11 \mathrm{~b}^{+}$Ly $6 \mathrm{C}^{\text {hi }}$ monocytes are recruited to the pancreas, where they differentiate from $\mathrm{CD} 11 \mathrm{~b}^{+} \mathrm{Ly} 6 \mathrm{C}^{\text {hi }} \mathrm{MHCII}{ }^{\text {hi }}$ cells into $\mathrm{CD}_{11 \mathrm{~b}}{ }^{\mathrm{Ly}} 6 \mathrm{C}^{\mathrm{lo}} \mathrm{MHCII}^{\mathrm{lo}} \mathrm{M} 2-$ like or tissue-resident macrophagelike cells. When $\mathrm{Ccr}^{-/-}$mice were used, though the CCL2/CCR2mediated Ly- $6 \mathrm{C}^{\text {hi }}$ monocyte recruitment was blocked, there was compensatory proliferation of $\mathrm{MHC}-\mathrm{II}^{\mathrm{lo}}$ tissue-resident macrophages and PDL did not significantly alter $\beta$ cell proliferation (42). Because CSF1R signaling reportedly mediates local macrophage replication and promotes differentiation of $\mathrm{MHC}-\mathrm{II}^{\mathrm{hi}}$ into M2-like MHC-II ${ }^{\mathrm{lo}}$ macrophages in general settings (112), the effect of CSF1R neutralization was analyzed in the PDL model. CSF1R neutralization not only inhibited tissue-resident macrophage proliferation but also reduced differentiation of recruited MHC-II ${ }^{\mathrm{hi}}$ macrophages into MHC-II ${ }^{\mathrm{lo}}$ macrophages (ref. 111 and Figure 2).

These reports demonstrate that both macrophages recruited from bone marrow and islet-resident macrophages exhibit a capacity to support $\beta$ cell proliferation through acquisition of an M2-like macrophage phenotype supported by CSF1R signaling. Furthermore, these studies suggest that multiple cytokines, including TGF- $\beta 1$, VEGFA, and CSF1, secreted from multiple cell types, including macrophages, $\beta$ cells, and quiescent endothelial cells, contribute to establishing a microenvironment that maintains the macrophage M2-like phenotype and $\beta$ cell proliferation (Figure 2).

\section{Conclusions}

In addition to the contribution of inflammation to insulin resistance, the existence of islet inflammation and its causative involvement in $\beta$ cell dysfunction in T2D is now well appreciated. Islet cells, including $\beta$ cells, play an important role in the initiation of islet inflammation, as they have the ability to sense stimuli and secrete chemokines as well as hIAPP to activate macrophages. Most studies indicate that macrophages are the main source of proinflammatory cytokines within islets. Among these proinflammatory cytokines, IL-1 $\beta$ secreted from M1-like macrophages plays a crucial role in the initiation and amplification of islet inflammation. In comparison, M2-like macrophages are indispensible for both islet development and $\beta$ cell proliferation in adults. Sterile factors stimulating macrophage activation, the origin of the macrophages, and the local milieu established by multiple cytokines from several cell types within islets are all key determinants of macrophage polarization and function in both normal physiology and T2D pathology.

The new understanding of $\beta$ cell failure in T2D gained from studies of islet inflammation provides clues to translational possibilities. For example, as inflammasome/IL-1 $\beta$ signaling is the most common and impactful pathway activated in islets of multiple T2D models, strategies targeting IL-1 signaling have produced encouraging results in clinical studies $(27,28,54,55)$. In addition, because the correct dosage of IL-1 $\beta$-neutralizing antibody is critical for recovery of $\beta$ cell function in humans, a greater understanding of the macrophages' role in islet physiology should help to optimize IL-1-targeted strategies. Given that inflammatory pathologic changes have been observed in only a portion of T2D patients $(12-14,18)$, the identification of biomarkers that correlate with islet inflammation will help to enhance the effectiveness of therapeutics that target islet inflammation by allowing for appropriate patient selection. Also, in order to avoid systemically suppressing the function of M1-like macrophages, targeting the mechanisms of macrophage M1-like activation that are unique to islets could potentially lead to safe and effective strategies for suppressing islet inflammation (Figure 3).

It would also be desirable to support processes related to the normal physiology and beneficial aspects of islet macrophage behavior, such as their facilitation of $\beta$ cell proliferation. The key components necessary to establish a physiological islet microenvironment remain unclear. The fact that a microenvironment supporting $\beta$ cell proliferation is established through complex communication among several cell types via multiple cytokines makes development of translational approaches difficult. However, CSF1 signaling appears to be a core component, as its importance has been demonstrated in multiple settings, including development, a pancreatitis model, and a PDL model. Furthermore, targeting $\beta$ cell proliferation may be effective in both T1D and T2D settings. Therefore, future studies of the beneficial aspects of islet macrophage biology could be highly productive (Figure 3).

An improved understanding of islet macrophage biology should enable development of strategies for blocking pathologic T2D islet inflammation to ameliorate $\beta$ cell dysfunction while promoting physiologic immune cell function to enhance $\beta$ cell proliferation, which may have broad translational implications (Figure 3).

\section{Acknowledgments}

We thank Alexander Bartelt, Kathryn C. Claiborn, Kacey Prentice, Lauren T. Robertson, and Scott B. Widenmaier for helpful discussions.

Address correspondence to: Kosei Eguchi, Department of Genetics and Complex Diseases and Sabri ülker Center, Harvard T.H. Chan School of Public Health, 677 Huntington Ave., Boston, Massachusetts 02115, USA. Phone: 617.432.1951; E-mail: eguchik-tky@umin.org. 
1. Hotamisligil GS, Shargill NS, Spiegelman BM. Adipose expression of tumor necrosis factoralpha: direct role in obesity-linked insulin resistance. Science. 1993;259(5091):87-91.

2. Hotamisligil GS. Inflammation and metabolic disorders. Nature. 2006;444(7121):860-867.

3. Olefsky JM, Glass CK. Macrophages, inflammation, and insulin resistance. Annu Rev Physiol. 2010;72:219-246.

4. Lumeng CN, Saltiel AR. Inflammatory links between obesity and metabolic disease. JClin Invest. 2011;121(6):2111-2117.

5. Kumar V, Abbas AK, Aster JC. Robbins Basic Pathology. 9th ed. Philadelphia, Pennsylvania, USA: Saunders; 2013.

6. Lumeng CN, Bodzin JL, Saltiel AR. Obesity induces a phenotypic switch in adipose tissue macrophage polarization. J Clin Invest. 2007;117(1):175-184.

7. Winer S, et al. Normalization of obesity-associated insulin resistance through immunotherapy. Nat Med. 2009;15(8):921-929.

8. Nishimura S, et al. CD8 ${ }^{+}$effector T cells contribute to macrophage recruitment and adipose tissue inflammation in obesity. Nat Med. 2009;15(8):914-920.

9. Grundy SM, et al. Diagnosis and management of the metabolic syndrome: an American Heart Association/National Heart, Lung, and Blood Institute Scientific Statement. Circulation. 2005;112(17):2735-2752.

10. Ferrannini E, Gastaldelli A, Miyazaki Y, Matsuda M, Mari A, DeFronzo RA. beta-Cell function in subjects spanning the range from normal glucose tolerance to overt diabetes: a new analysis. JClin Endocrinol Metab. 2005;90(1):493-500.

11. Mari A, et al. Impaired $\beta$ cell glucose sensitivity rather than inadequate compensation for insulin resistance is the dominant defect in glucose intolerance. Diabetologia. 2010;53(4):749-756.

12. Ehses JA, et al. Increased number of islet-associated macrophages in type 2 diabetes. Diabetes. 2007;56(9):2356-2370.

13. Richardson SJ, Willcox A, Bone AJ, Foulis AK, Morgan NG. Islet-associated macrophages in type 2 diabetes. Diabetologia. 2009;52(8):1686-1688.

14. Kamata K, et al. Islet amyloid with macrophage migration correlates with augmented $\beta$-cell deficits in type 2 diabetic patients. Amyloid. 2014;21(3):191-201.

15. Butcher MJ, et al. Association of proinflammatory cytokines and islet resident leucocytes with islet dysfunction in type 2 diabetes. Diabetologia. 2014;57(3):491-501.

16. Martino L, et al. Mast cells infiltrate pancreatic islets in human type 1 diabetes. Diabetologia. 2015;58(11):2554-2562.

17. Westermark P. Quantitative studies on amyloid in the islets of Langerhans. Ups J Med Sci. 1972;77(2):91-94.

18. Zhao HL, et al. Prevalence and clinicopathological characteristics of islet amyloid in chinese patients with type 2 diabetes. Diabetes. 2003;52(11):2759-2766.

19. Hayden MR. Islet amyloid and fibrosis in the cardiometabolic syndrome and type 2 diabetes mellitus. J Cardiometab Syndr. 2007;2(1):70-75.

20. Masters SL, et al. Activation of the NLRP3 inflam- masome by islet amyloid polypeptide provides a mechanism for enhanced IL-1 $\beta$ in type 2 diabetes. Nat Immunol. 2010;11(10):897-904.

21. Eguchi K, et al. Saturated fatty acid and TLR signaling link $\beta$ cell dysfunction and islet inflammation. Cell Metab. 2012;15(4):518-533.

22. Jourdan T, et al. Activation of the Nlrp3 inflammasome in infiltrating macrophages by endocannabinoids mediates beta cell loss in type 2 diabetes. Nat Med. 2013;19(9):1132-1140.

23. Westwell-Roper CY, Ehses JA, Verchere CB. Resident macrophages mediate islet amyloid polypeptide-induced islet IL-1 $\beta$ production and $\beta$-cell dysfunction. Diabetes. 2014;63(5):1698-1711.

24. Ehses JA, et al. IL-1 antagonism reduces hyperglycemia and tissue inflammation in the type 2 diabetic GK rat. Proc Natl Acad Sci U S A. 2009;106(33):13998-14003.

25. Sauter NS, Schulthess FT, Galasso R, Castellani LW, Maedler K. The antiinflammatory cytokine interleukin-1 receptor antagonist protects from high-fat diet-induced hyperglycemia. Endocrinology. 2008;149(5):2208-2218.

26. Larsen CM, et al. Interleukin-1-receptor antagonist in type 2 diabetes mellitus. $N$ Engl JMed. 2007;356(15):1517-1526.

27. Cavelti-Weder C, et al. Effects of gevokizumab on glycemia and inflammatory markers in type 2 diabetes. Diabetes Care. 2012;35(8):1654-1662.

28. Rissanen A, Howard CP, Botha J, Thuren T, Global I. Effect of anti-IL-1 $\beta$ antibody (canakinumab) on insulin secretion rates in impaired glucose tolerance or type 2 diabetes: results of a randomized, placebo-controlled trial. Diabetes Obes Metab. 2012;14(12):1088-1096.

29. Rodriguez-Calvo T, Ekwall O, Amirian N, Zapardiel-Gonzalo J, von Herrath MG. Increased immune cell infiltration of the exocrine pancreas: a possible contribution to the pathogenesis of type 1 diabetes. Diabetes. 2014;63(11):3880-3890.

30. Marchetti P. Islet inflammation in type 2 diabetes. Diabetologia. 2016;59(4):668-672.

31. Brooks-Worrell BM, Boyko EJ, Palmer JP. Impact of islet autoimmunity on the progressive $\beta$-cell functional decline in type 2 diabetes. Diabetes Care. 2014;37(12):3286-3293.

32. Davies LC, Jenkins SJ, Allen JE, Taylor PR. Tissue-resident macrophages. Nat Immunol. 2013;14(10):986-995

33. Gosselin D, et al. Environment drives selection and function of enhancers controlling tissue-specific macrophage identities. Cell. 2014;159(6):1327-1340.

34. Lavin Y, et al. Tissue-resident macrophage enhancer landscapes are shaped by the local microenvironment. Cell. 2014;159(6):1312-1326.

35. Mantovani A, Sica A, Locati M. Macrophage polarization comes of age. Immunity. 2005;23(4):344-346.

36. Murray PJ, Wynn TA. Protective and pathogenic functions of macrophage subsets. Nat Rev Immunol. 2011;11(11):723-737.

37. Lech M, Anders HJ. Macrophages and fibrosis: How resident and infiltrating mononuclear phagocytes orchestrate all phases of tissue injury and repair. Biochim Biophys Acta. 2013;1832(7):989-997.
38. Kelly J, Ali Khan A, Yin J, Ferguson TA, Apte RS. Senescence regulates macrophage activation and angiogenic fate at sites of tissue injury in mice. J Clin Invest. 2007;117(11):3421-3426.

39. Geutskens SB, Otonkoski T, Pulkkinen MA, Drexhage HA, Leenen PJ. Macrophages in the murine pancreas and their involvement in fetal endocrine development in vitro. J Leukoc Biol. 2005;78(4):845-852.

40. Banaei-Bouchareb L, et al. Insulin cell mass is altered in Csflop/Csflop macrophage-deficient mice. J Leukoc Biol. 2004;76(2):359-367.

41. Tessem JS, et al. Critical roles for macrophages in islet angiogenesis and maintenance during pancreatic degeneration. Diabetes. 2008;57(6):1605-1617.

42. Xiao X, et al. M2 macrophages promote beta-cel proliferation by up-regulation of SMAD7. Proc Natl Acad Sci U S A. 2014;111(13):E1211-E1220.

43. Criscimanna A, Coudriet GM, Gittes GK, Piganelli JD, Esni F. Activated macrophages create lineage-specific microenvironments for pancreatic acinar- and $\beta$-cell regeneration in mice. Gastroenterology. 2014;147(5):1106-18.e11.

44. Cao X, Han ZB, Zhao H, Liu Q. Transplantation of mesenchymal stem cells recruits trophic macrophages to induce pancreatic beta cell regeneration in diabetic mice. Int J Biochem Cell Biol. 2014;53:372-379.

45. Homo-Delarche F, et al. Islet inflammation and fibrosis in a spontaneous model of type 2 diabetes, the GK rat. Diabetes. 2006;55(6):1625-1633.

46. Igoillo-Esteve $\mathrm{M}$, et al. Palmitate induces a pro-inflammatory response in human pancreatic islets that mimics CCL2 expression by beta cells in type 2 diabetes. Diabetologia. 2010;53(7):1395-1405.

47. Atkinson MA, Maclaren NK. The pathogenesis of insulin-dependent diabetes mellitus. $\mathrm{N} \mathrm{Engl} \mathrm{J}$ Med.1994;331(21):1428-1436.

48. Wallberg M, Cooke A. Immune mechanisms in type 1 diabetes. Trends Immunol. 2013;34(12):583-591.

49. Maedler K, et al. Glucose-induced beta cell production of IL-1beta contributes to glucotoxicity in human pancreatic islets. JClin Invest. 2002;110(6):851-860.

50. Zhou R, Tardivel A, Thorens B, Choi I, Tschopp J. Thioredoxin-interacting protein links oxidative stress to inflammasome activation. Nat Immunol. 2010;11(2):136-140.

51. Ikeda H. KK mouse. Diabetes Res Clin Pract. 1994;24(suppl):S313-S316.

52. Cucak H, Grunnet LG, Rosendahl A. Accumulation of M1-like macrophages in type 2 diabetic islets is followed by a systemic shift in macrophage polarization. JLeukoc Biol. 2014;95(1):149-160.

53. Calderon B, et al. The pancreas anatomy conditions the origin and properties of resident macrophages. J Exp Med. 2015;212(10):1497-1512.

54. van Asseldonk EJ, Stienstra R, Koenen TB, Joosten LA, Netea MG, Tack CJ. Treatment with Anakinra improves disposition index but not insulin sensitivity in nondiabetic subjects with the metabolic syndrome: a randomized, doubleblind, placebo-controlled study. JClin Endocrinol Metab. 2011;96(7):2119-2126.

55. Sloan-Lancaster J, et al. Double-blind, ran- 
domized study evaluating the glycemic and anti-inflammatory effects of subcutaneous LY2189102, a neutralizing IL-1 $\beta$ antibody, in patients with type 2 diabetes. Diabetes Care. 2013;36(8):2239-2246.

56. Donath MY. Targeting inflammation in the treatment of type 2 diabetes: time to start. Nat Rev Drug Discov. 2014;13(6):465-476.

57. Herder C, Dalmas E, Böni-Schnetzler M, Donath MY. The IL-1 pathway in type 2 diabetes and cardiovascular complications. Trends Endocrinol Metab. 2015;26(10):551-563.

58. Shi H, Kokoeva MV, Inouye K, Tzameli I, Yin H, Flier JS. TLR4 links innate immunity and fatty acid-induced insulin resistance. J Clin Invest . 2006;116(11):3015-3025.

59. Jia L, et al. Hepatocyte Toll-like receptor 4 regulates obesity-induced inflammation and insulin resistance. Nat Commun. 2014;5:3878.

60. Vandanmagsar B, et al. The NLRP3 inflammasome instigates obesity-induced inflammation and insulin resistance. Nat Med. 2011;17(2):179-188.

61. Kono H, Rock KL. How dying cells alert the immune system to danger. Nat Rev Immunol. 2008;8(4):279-289.

62. Vogl T, et al. Mrp8 and Mrp14 are endogenous activators of Toll-like receptor 4, promoting lethal, endotoxin-induced shock. Nat Med. 2007;13(9):1042-1049.

63. Klune JR, Dhupar R, Cardinal J, Billiar TR, Tsung A. HMGB1: endogenous danger signaling. Mol Med. 2008;14(7-8):476-484.

64. Eguchi K, Manabe I. Toll-like receptor, lipotoxicity and chronic inflammation: the pathological link between obesity and cardiometabolic disease. J Atheroscler Thromb. 2014;21(7):629-639.

65. Krüger B, et al. Islet-expressed TLR2 and TLR4 sense injury and mediate early graft failure after transplantation. Eur JImmunol. 2010;40(10):2914-2924.

66. Ehses JA, et al. Toll-like receptor 2-deficient mice are protected from insulin resistance and beta cell dysfunction induced by a high-fat diet. Diabetologia. 2010;53(8):1795-1806.

67. Böni-Schnetzler M, et al. Free fatty acids induce a proinflammatory response in islets via the abundantly expressed interleukin-1 receptor I. Endocrinology. 2009;150(12):5218-5229.

68. Maloy AL, Longnecker DS, Greenberg ER. The relation of islet amyloid to the clinical type of diabetes. Hum Pathol.1981;12(10):917-922.

69. Cooper GJ, Willis AC, Clark A, Turner RC, Sim $\mathrm{RB}$, Reid KB. Purification and characterization of a peptide from amyloid-rich pancreases of type 2 diabetic patients. Proc Natl Acad Sci U S A. 1987;84(23):8628-8632.

70. Westermark P, Wernstedt C, Wilander E, Hayden DW, O'Brien TD, Johnson KH. Amyloid fibrils in human insulinoma and islets of Langerhans of the diabetic cat are derived from a neuropeptide-like protein also present in normal islet cells. Proc Natl Acad Sci U S A. 1987;84(11):3881-3885.

71. Ogawa A, Harris V, McCorkle SK, Unger RH, Luskey KL. Amylin secretion from the rat pancreas and its selective loss after streptozotocin treatment. J Clin Invest. 1990;85(3):973-976.

72. Wei L, et al. The molecular basis of distinct aggre- gation pathways of islet amyloid polypeptide. J Biol Chem. 2011;286(8):6291-6300.

73. Buchanan LE, et al. Mechanism of IAPP amyloid fibril formation involves an intermediate with a transient $\beta$-sheet. Proc Natl Acad Sci U S A. 2013;110(48):19285-19290.

74. Lorenzo A, Razzaboni B, Weir GC, Yankner BA. Pancreatic islet cell toxicity of amylin associated with type-2 diabetes mellitus. Nature. 1994;368(6473):756-760.

75. Haataja L, Gurlo T, Huang CJ, Butler PC. Islet amyloid in type 2 diabetes, and the toxic oligomer hypothesis. Endocr Rev. 2008;29(3):303-316.

76. Green J, et al. Full-length rat amylin forms fibrils following substitution of single residues from human amylin. J Mol Biol. 2003;326(4):1147-1156.

77. Fox N, et al. Human islet amyloid polypeptide transgenic mice as a model of non-insulindependent diabetes mellitus (NIDDM). FEBS Lett.1993;323(1-2):40-44.

78. de Koning EJ, et al. Human islet amyloid polypeptide accumulates at similar sites in islets of transgenic mice and humans. Diabetes. 1994;43(5):640-644.

79. Hull RL, et al. Increased dietary fat promotes islet amyloid formation and beta-cell secretory dysfunction in a transgenic mouse model of islet amyloid. Diabetes. 2003;52(2):372-379.

80. Meier DT, Morcos M, Samarasekera T, Zraika S, Hull RL, Kahn SE. Islet amyloid formation is an important determinant for inducing islet inflammation in high-fat-fed human IAPP transgenic mice. Diabetologia. 2014;57(9):1884-1888.

81. Westwell-Roper C, et al. IL-1 blockade attenuates islet amyloid polypeptide-induced proinflammatory cytokine release and pancreatic islet graft dysfunction. J Immunol. 2011;187(5):2755-2765.

82. Westwell-Roper C, Denroche HC, Ehses JA, Verchere CB. Differential activation of innate immune pathways by distinct islet amyloid polypeptide (IAPP) aggregates. J Biol Chem. 2016;291(17):8908-8917.

83. Westwell-Roper CY, Chehroudi CA, Denroche HC, Courtade JA, Ehses JA, Verchere CB. IL-1 mediates amyloid-associated islet dysfunction and inflammation in human islet amyloid polypeptide transgenic mice. Diabetologia. 2015;58(3):575-585.

84. de Koning EJ, et al. Macrophages and pancreatic islet amyloidosis. Amyloid. 1998;5(4):247-254.

85. Halle A, et al. The NALP3 inflammasome is involved in the innate immune response to amyloid-beta. Nat Immunol. 2008;9(8):857-865.

86. Heneka MT, et al. NLRP3 is activated in Alzheimer's disease and contributes to pathology in APP/ PS1 mice. Nature. 2013;493(7434):674-678.

87. Badman MK, Pryce RA, Chargé SB, Morris JF, Clark A. Fibrillar islet amyloid polypeptide (amylin) is internalised by macrophages but resists proteolytic degradation. Cell Tissue Res. 1998;291(2):285-294

88. Bauernfeind FG, et al. Cutting edge: NF-kappaB activating pattern recognition and cytokine receptors license NLRP3 inflammasome activation by regulating NLRP3 expression. J Immunol.
2009;183(2):787-791.

89. Miller YI, Viriyakosol S, Binder CJ, Feramisco JR, Kirkland TN, Witztum JL. Minimally modified LDL binds to CD14, induces macrophage spreading via TLR4/MD-2, and inhibits phagocytosis of apoptotic cells. J Biol Chem. 2003;278(3):1561-1568.

90. Abderrahmani A, et al. Human high-density lipoprotein particles prevent activation of the JNK pathway induced by human oxidised low-density lipoprotein particles in pancreatic beta cells. Diabetologia. 2007;50(6):1304-1314.

91. Hutton MJ, Westwell-Roper C, Soukhatcheva G, Plesner A, Dutz JP, Verchere CB. Islet allograft rejection is independent of toll-like receptor signaling in mice. Transplantation. 2009;88(9):1075-1080.

92. Wen H, et al. Fatty acid-induced NLRP3-ASC inflammasome activation interferes with insulin signaling. Nat Immunol. 2011;12(5):408-415.

93. Pacher P, Bátkai S, Kunos G. The endocannabinoid system as an emerging target of pharmacotherapy. Pharmacol Rev. 2006;58(3):389-462.

94. Maccarrone M, et al. Endocannabinoid signaling at the periphery: 50 years after THC. Trends Pharmacol Sci. 2015;36(5):277-296.

95. Rohrbach K, et al. Ibipinabant attenuates $\beta$-cell loss in male Zucker diabetic fatty rats independently of its effects on body weight. Diabetes Obes Metab. 2012;14(6):555-564.

96. Tam J, et al. Peripheral cannabinoid-1 receptor inverse agonism reduces obesity by reversing leptin resistance. Cell Metab. 2012;16(2):167-179.

97. Maedler K, et al. Low concentration of interleukin-1beta induces FLICE-inhibitory proteinmediated $\beta$-cell proliferation in human pancreatic islets. Diabetes. 2006;55(10):2713-2722.

98. Hajmrle C, et al. Interleukin-1 signaling contributes to acute islet compensation. JCI Insight. 2016;1(4):e86055.

99. Hume DA, Robinson AP, MacPherson GG, Gordon S. The mononuclear phagocyte system of the mouse defined by immunohistochemical localization of antigen F4/80. J Exp Med. 1983;158(5):1522-1536.

100.Banaei-Bouchareb L, Peuchmaur M, Czernichow P, Polak M. A transient microenvironment loaded mainly with macrophages in the early developing human pancreas. JEndocrinol. 2006; 188(3):467-480.

101.Schulz C, et al. A lineage of myeloid cells independent of $\mathrm{Myb}$ and hematopoietic stem cells. Science. 2012;336(6077):86-90.

102. Gomez Perdiguero E, et al. Tissue-resident macrophages originate from yolk-sacderived erythro-myeloid progenitors. Nature. 2015;518(7540):547-551.

103. Bain CC, et al. Constant replenishment from circulating monocytes maintains the macrophage pool in the intestine of adult mice. Nat Immunol. 2014;15(10):929-937.

104.Eguchi K, Manabe I. Macrophages and islet inflammation in type 2 diabetes. Diabetes Obes Metab. 2013;15 Suppl 3:152-158.

105. Morris DL. Minireview: Emerging concepts in islet macrophage biology in type 2 diabetes. $M o l$ Endocrinol. 2015;29(7):946-962.

106.Xiao X, et al. TGF $\beta$ receptor signaling is essen- 
tial for inflammation-induced but not $\beta$-cell workload-induced $\beta$-cell proliferation. Diabetes. 2013;62(4):1217-1226.

107. Brissova M, et al. Pancreatic islet production of vascular endothelial growth factor $-\mathrm{a}$ is essential for islet vascularization, revascularization, and function. Diabetes. 2006;55(11):2974-2985.

108. Agudo J, et al. Vascular endothelial growth factor-mediated islet hypervascularization and inflammation contribute to progressive reduction of $\beta$-cell mass. Diabetes. 2012;61(11):2851-2861.

109. Brissova M, et al. Islet microenvironment, modulated by vascular endothelial growth factor-A signaling, promotes $\beta$ cell regeneration. Cell Metab. 2014;19(3):498-511.

110. He H, et al. Endothelial cells provide an instructive niche for the differentiation and functional polarization of M2-like macrophages. Blood. 2012;120(15):3152-3162.

111. Van Gassen N, et al. Macrophage dynamics are regulated by local macrophage proliferation and monocyte recruitment in injured pancreas. Eur J Immunol. 2015;45(5):1482-1493.

112. Hamilton JA. Colony-stimulating factors in inflammation and autoimmunity. Nat Rev Immunol. 2008;8(7):533-544. 